\title{
Comparison of Endoscopic Ultrasound-Guided Tissue Acquisition Using a 20-Gauge Menghini Needle with a Lateral Forward Bevel and a 22-Gauge Franseen Needle: A Single-Center Large Cohort Study
}

\author{
Takafumi Mie, Takashi Sasaki, Ryo Kanata, Takaaki Furukawa, Tsuyoshi Takeda, Akiyoshi Kasuga, Masato Matsuyama, Masato \\ Ozaka and Naoki Sasahira \\ Department of Hepato-Biliary-Pancreatic Medicine, Cancer Institute Hospital of Japanese Foundation for Cancer Research, Tokyo, \\ Japan
}

Background/Aims: Several fine-needle biopsy (FNB) needles are available for endoscopic ultrasound (EUS)-guided tissue acquisition. However, there is disagreement on which type of needle has the best diagnostic yield. The aim of this study was to compare the performance and safety of two commonly used EUS-FNB needles.

Methods: We retrospectively analyzed consecutive patients who underwent EUS-FNB between June 2016 and March 2020 in our hospital. Two types of needles were evaluated: a 20-gauge Menghini needle with a lateral forward bevel and a 22-gauge Franseen needle. Rapid on-site evaluation was performed in all the cases. A multivariate analysis was performed to clarify the negative predictive factors for obtaining a histological diagnosis. Propensity score matching was performed to compare the diagnostic yields of these two needles.

Results: We analyzed 666 patients and 690 lesions. The overall diagnostic rate of histology alone was $88.8 \%$, and the overall adverse event rate was $1.5 \%$. Transduodenal access and small lesions $(\leq 2 \mathrm{~cm})$ were identified as negative predictive factors for obtaining a histological diagnosis. After propensity score matching, 482 lesions were analyzed. The diagnostic accuracy rates of histology in the $\mathrm{M}$ and $\mathrm{F}$ needle groups were $89.2 \%$ and $88.8 \%$, respectively $(p=1.00)$.

Conclusions: Both the needles showed high diagnostic yield, and no significant difference in performance was observed between the two. Clin Endosc 2021;54:730-738

Key Words: Biopsy; Endoscopic ultrasonography; Endoscopic ultrasound-guided fine-needle aspiration

\section{INTRODUCTION}

Endoscopic ultrasound (EUS)-guided tissue acquisition is widely used for the pathological diagnosis of various lesions, including pancreatic lesions, submucosal lesions, and lymph

Received: September 14, 2020 Revised: October 31, 2020

Accepted: November 8, 2020

Correspondence: Takashi Sasaki

Department of Hepato-Biliary-Pancreatic Medicine, Cancer Institute Hospital of Japanese Foundation for Cancer Research, 3-8-31 Ariake, Koto, Tokyo 135-8550, Japan

Tel: +81-3-3520-0111, Fax: +81-3-3520-0141, E-mail: sasakit-tky@umin.ac.jp ORCID: https://orcid.org/0000-0001-7109-9835

(c) This is an Open Access article distributed under the terms of the Creative Commons Attribution Non-Commercial License (http://creativecommons.org/ licenses/by-nc/3.0) which permits unrestricted non-commercial use, distribution, and reproduction in any medium, provided the original work is properly cited. node lesions. ${ }^{1-3}$ In the past, EUS-fine-needle aspiration (FNA) has been performed, and its main purpose was to diagnose whether a tumor was benign or malignant. However, the diagnostic ability of histology through this method is approximately $70 \%$, and immunostaining is often challenging. ${ }^{4}$ Thereafter, a 19-gauge $(G)$ needle and a Tru-Cut needle were used to enable better tissue sampling. However, handling these needles was difficult due to their stiffness. ${ }^{5}$ Therefore, further improvements in the biopsy needle became necessary.

EUS-fine-needle biopsy (FNB) needles were developed to obtain more tissue, and several FNB needles are now commercially available. An EchoTip ProCore ${ }^{\circledR}$ HD Ultrasound Biopsy Needle (Cook Endoscopy Inc., Limerick, Ireland), which had a side hole with a reverse bevel, was developed. However, previous meta-analyses have shown no differences in the efficiency of tissue acquisition between this FNB needle and the 
conventional FNA needle. ${ }^{6-8}$ To improve the amount of tissue obtained and the histological diagnostic yield, a 20 G EchoTip ProCore ${ }^{\circledR}$ HD Ultrasound Biopsy Needle (Cook Endoscopy Inc.) was developed, which had a side hole with a forward bevel, and there are studies reporting its advantages. ${ }^{9-12}$ This needle is reported to obtain adequate samples for histology in approximately $92 \%$ of cases. ${ }^{9,12,13}$ On the other hand, an Acquire ${ }^{\mathrm{TM}}$ Endoscopic Ultrasound Fine-Needle Biopsy Device (Boston Scientific Corporation, Marlborough, MA, USA) was developed which has a unique needle tip with three points. This needle is reported to have a high probability $(96 \%-97 \%)$ of obtaining a sufficient amount of tissue for histological diagnosis. ${ }^{14,15}$ In addition, a SharkCore ${ }^{\mathrm{TM}}$ FNB Exchange System (Covidien/Medtronic, Whiteley, UK) is used worldwide. This needle has a special tip shape, and a high acquisition rate for histological diagnosis has been reported (95\%). ${ }^{16}$

Recently, several FNB needles have been reported to be superior to FNA needles in prospective randomized studies. ${ }^{17-20}$ The samples collected with FNB needles have been used for immunostaining and genetic testing. ${ }^{21-23}$ However, there is disagreement on which FNB needle leads to the best diagnostic yield. Additionally, the type of FNB needle suitable for different lesions, access routes, and for small lesions remains unclear. Therefore, we conducted a large cohort study to evaluate the diagnostic yield and the safety associated with two EUSFNB needles.

\section{PATIENTS AND METHODS}

We retrospectively analyzed consecutive patients who underwent EUS-FNB at our institution between June 2016 and March 2020. Two types of FNB needles were predominantly used during the study period: (1) A 20 G EchoTip ProCore ${ }^{\circledR}$ HD Ultrasound Biopsy Needle (Cook Endoscopy Inc.), an FNB Menghini (M) needle that has a core trap with a lateral forward bevel (Fig. 1A) and (2) A 22 G Acquire ${ }^{\mathrm{TM}}$ Endoscopic Ultrasound Fine-Needle Biopsy (Boston Scientific Corporation), an FNB Franseen (F) needle (Fig. 1B). The following patients were excluded: (1) Those who underwent EUS-FNB for one lesion with several types of needles, and did not receive an individual diagnosis for the samples acquired with each needle, and (2) those who could not be followed up until the final diagnosis.

\section{Procedure}

EUS-guided tissue acquisition was performed using a convex linear-array echoendoscope (GF-UCT260; Olympus Medical Systems, Tokyo, Japan) under moderate sedation with midazolam and analgesia with pethidine. The use of each EUS-FNB needle was divided over time: $\mathrm{M}$ needle was used between June 2016 and January 2019 and F needle between February 2019 and March 2020. The procedure was performed by experts ( $\geq 5$ years of experience in EUS tissue acquisition) or trainees ( $<5$ years of experience in EUS tissue acquisition) under the guidance of experts.

The size of the lesion was defined as the maximum diameter visualized on EUS, and the length of the FNB needle passing through the lesion was defined as the puncture length. At the first puncture, a $10 \mathrm{~mL}$ syringe suction was applied, and 20 strokes were performed using the fanning technique. ${ }^{24}$ When large amounts of blood were suctioned, the operator decided to use the slow pull method for the subsequent session. ${ }^{25}$ After each puncture, a rapid on-site evaluation (ROSE) was performed by the cytologist. On confirming that the target tissue was collected for ROSE, the procedure was completed with one additional puncture to obtain a sufficient sample for histological diagnosis, provided the lesion could be safely punctured again. Finally, the obtained specimens were fixed in $7 \%$ formalin and processed for histological examination.

\section{Final diagnosis}

For surgical cases, the final diagnosis was made using the resected specimen. For unresectable lesions, the final diagnosis was made by a combination of cytology or histology of the samples obtained by EUS-FNB and the subsequent clinical follow-ups for at least six months.

\section{Evaluation}

This study evaluated the diagnostic yield and the adverse events (AEs) associated with EUS-FNB. The diagnostic yield was evaluated per lesion, while AEs were calculated per patient. When the same needle was used to puncture multiple lesions, each lesion was evaluated individually. If two types of needles were used for one lesion, the diagnostic yield was evaluated for each needle. The tissue acquisition rate was defined as the sum of lesions in which a histological diagnosis could be obtained, divided by the total number of lesions. An accurate diagnosis was defined as a malignant disease being diagnosed as malignant and a benign disease as benign. Diagnostic accuracy was defined as the sum of accurate diagnoses divided by the total number of lesions. AEs were defined as any postprocedural events using a lexicon for endoscopic AEs. ${ }^{26}$

\section{Statistical analysis}

Continuous variables were presented as medians (ranges) and compared using the Mann-Whitney $U$ test. Categorical variables were described as absolute numbers (proportions) 


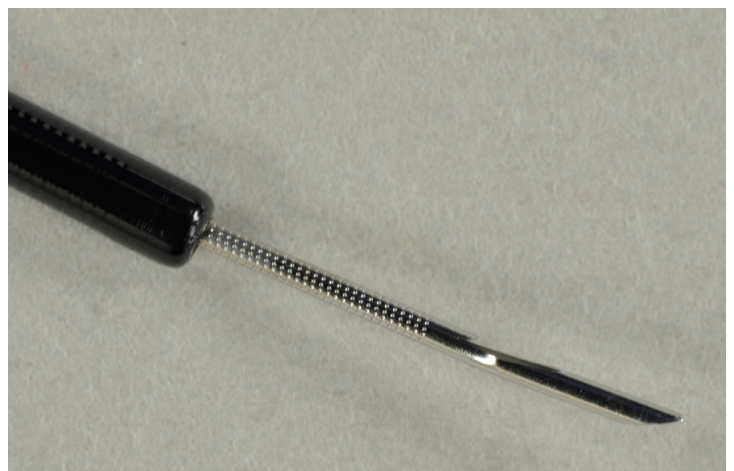

(A)

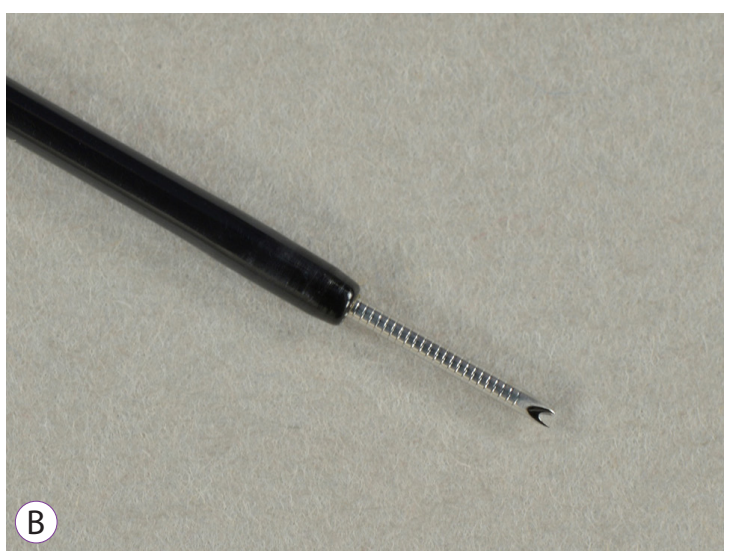

Fig. 1. The shape of the needle tips used in the study. (A) A 20-gauge EchoTip ProCore ${ }^{\circledR}$ HD Ultrasound Biopsy Needle (Cook Endoscopy Inc., Limerick, Ireland), an fine-needle biopsy (FNB) Menghini needle that has a core trap with a lateral forward bevel. (B) A 22 G Acquire ${ }^{\text {TM }}$ Endoscopic Ultrasound Fine-Needle Biopsy (Boston Scientific Corporation, Marlborough, MA, USA), an FNB Franseen needle.

and analyzed using the Chi-squared or Fisher's exact test. A $p$-value $<0.05$ was considered statistically significant. The following five variables were assessed by univariate analysis to identify the influencing factors for obtaining a histological diagnosis: FNB needle (M vs. F), location (pancreas vs. lymph node vs. submucosal tumor vs. others), access route (transgastric vs. transduodenal vs. others), size of lesion (small lesion; $\leq 2 \mathrm{~cm}$ vs. large lesion; $>2 \mathrm{~cm}$ ), and the physician performing the procedure (expert vs trainee). Factors in the univariate analysis with a $p$-value $<0.15$ were subjected to multivariate logistic regression analysis, and odds ratios (ORs) with a $95 \%$ confidence interval (CI) were calculated. Propensity score matching in a one-to-one ratio with a caliper width equal to 0.2 was applied to create comparable cohorts between the M and $\mathrm{F}$ groups. The propensity score was calculated with factors including the location of the lesion, access route, size of the lesion, puncture length, and number of punctures. All statistical analyses were performed using EZR ver. 1.40 (Saitama Medical Center, Jichi Medical University, Saitama, Japan). ${ }^{27}$ The clinical data were followed up until April 2020.

This study was approved by the ethics committee of our institution (approval number: 2019-1191). All procedures were performed in accordance with the Declaration of Helsinki and written informed consent for the procedure was obtained from all patients.

\section{RESULTS}

A total of 711 patients underwent EUS-FNB during this period. Out of these, 41 patients had EUS-FNB performed by several types of needles in one session and did not receive individual diagnoses for the samples obtained by each needle. Four patients were lost to follow-up. The above patients were excluded according to the exclusion criteria. Consequently, 666 patients and 690 lesions were analyzed in this study (Fig. 2). The final diagnoses included 614 malignant and 76 benign lesions. Fifteen patients received punctures for two or three lesions, and eight lesions had to be punctured by two FNB needles. M needle could puncture all the lesions successfully, while two lesions (a gastric submucosal tumor and an abdominal lymph node) could not be punctured by F needle (technical success rate; M 100\% [409/409] and F 99.3\% [279/281] $[p=0.17])$.

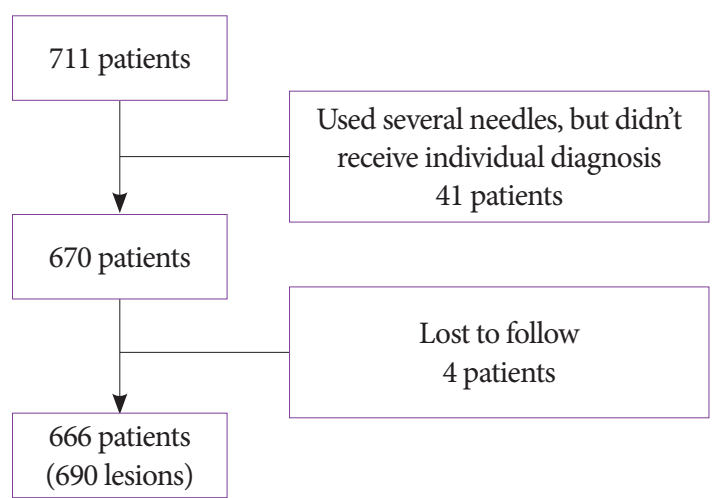

Fig. 2. Patient flow chart. Fifteen patients were punctured for two or three lesions, and eight lesions were punctured by two fine-needle biopsy needles. 
M needles were used for 409 lesions, and F needles for 281 lesions (Table 1). Pancreatic lesions were the most common $(77.2 \%, 533 / 690)$. There was no significant difference in the location of the lesion between the two groups $(p=0.10)$. Transgastric access was most frequently used in the $\mathrm{M}$ group $(p<0.01)$. The median size of the lesion was significantly larger, and the puncture length was significantly longer in the $M$ group (median size: M/F 27/25 mm; $p<0.01$; median puncture length: $M / F 21 / 18 \mathrm{~mm} ; p<0.01)$. There was no significant difference in the number of punctures between the two groups (median of 2 in both groups, $p=0.07$ ). There was no difference in the proportion of procedures performed by trainees be- tween the two groups $(p=0.11)$. The overall tissue acquisition rate was $98.4 \%$, and the diagnostic rates of histology alone and histology with cytology were $88.8 \%$ and $95.7 \%$, respectively. The tissue acquisition rates in the $\mathrm{M}$ and $\mathrm{F}$ groups were $99.8 \%$ and $96.4 \%(p<0.01)$, the diagnostic yields of histology alone in the $\mathrm{M}$ and $\mathrm{F}$ groups were $90.5 \%$ and $86.5 \%(p=0.11)$, and those of histology combined with cytology were $97.6 \%$ and 92.9\% ( $p<0.01)$, respectively.

Ten AEs occurred in total (1.5\%; 10/666). Five cases of mild bleeding, one case of mild pancreatitis, and one case of needle fracture occurred in the M group. ${ }^{28}$ Two cases of mild bleeding and two of mild pancreatitis occurred in the F group (M/F:

Table 1. Characteristic of Study Lesions in Each Needle $(n=690)$

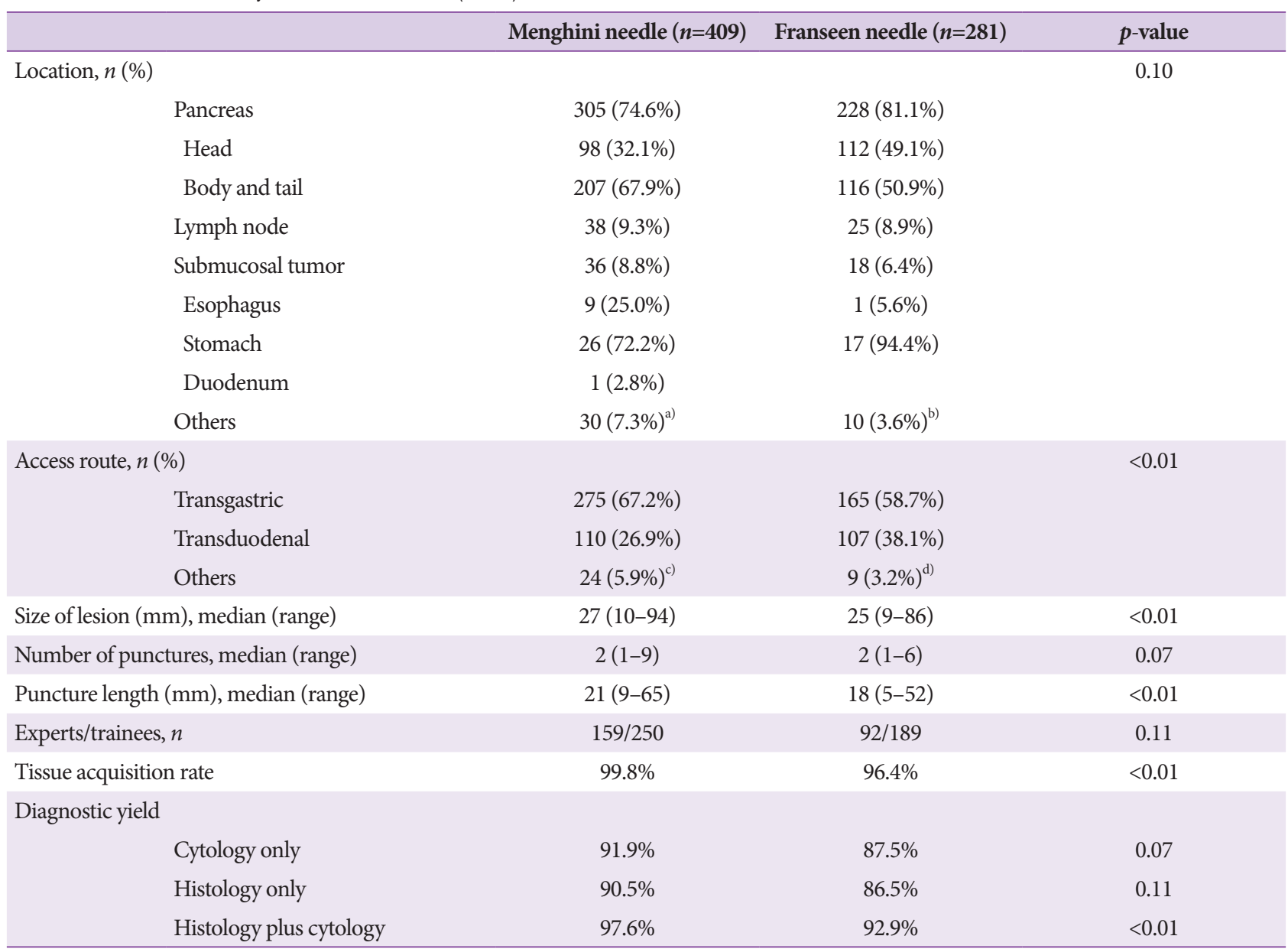

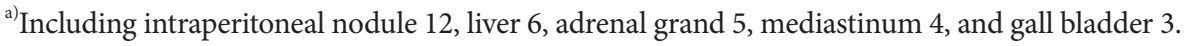

${ }^{b)}$ Including intraperitoneal nodule 8, liver 1, and mediastinum 1.

c) Transesophageal 24.

d) Transesophageal 4, through the jejunum 3, and through the rectum 2. 
$1.5 \% / 1.5 \%, p=1.00)$. Table 2 shows the multivariate analysis of factors influencing the diagnostic accuracy of histology. Multivariate logistic regression analysis revealed that transduodenal access and small lesions $(\leq 2 \mathrm{~cm})$ were independent negative predictive factors for obtaining a histological diagnosis (transduodenal access: OR, 0.50; 95\% CI, 0.31-0.82; $p<0.01$, small lesion: OR, 0.52; 95\% CI, 0.31-0.86; $p=0.01)$. The histological diagnosis rates of transduodenal access and small lesions were $83.9 \%$ (182/217) and 83.9\% (161/192), respectively. The diagnostic rates of histology combined with cytology of transduodenal access and small lesions were 94.9\% (206/217) and 91.1\% (175/192), respectively.

After the propensity score matching was performed, 482 lesions were extracted (Table 3 ). As a result, the $\mathrm{M}$ and $\mathrm{F}$ groups showed no differences in terms of location, access route, lesion size, puncture number, or puncture length. A higher number of procedures were performed by experts in the $\mathrm{M}$ group $(p=0.06)$. The results were similar in the two groups; the tissue acquisition rates in the $\mathrm{M}$ and $\mathrm{F}$ groups were $99.6 \%$ and $97.5 \%$ $(p=0.12)$, and the diagnostic yields of histology alone in the $\mathrm{M}$ and $\mathrm{F}$ groups were $89.2 \%$ and $88.8 \%(p=1.00)$ and those of histology plus cytology were $97.5 \%$ and $94.2 \%(p=0.11)$, respectively. The forest plot shows the subgroup analysis of the difference between the two needles regarding the diagnosis rate of histology alone (Fig. 3). There was no difference between the two groups. However, for lymph nodes, $\mathrm{M}$ needle tended to be better for obtaining a histological diagnosis (OR, 1.24 ; 95\% CI, $1.00-1.52 ; p=0.11$ ).

Table 2. Factors Influencing Histological Diagnostic Accuracy for Malignancy; Multivariate Analysis

\begin{tabular}{|c|c|c|c|c|}
\hline & \multicolumn{2}{|c|}{ Univariate } & \multicolumn{2}{|c|}{ Multivariate } \\
\hline & OR $(95 \% \mathrm{CI})$ & $p$-value & OR $(95 \% \mathrm{CI})$ & $p$-value \\
\hline \multicolumn{5}{|l|}{ FNB needle } \\
\hline Menghini needle & $1.48(0.92-2.39)$ & 0.10 & $1.24(0.76-2.02)$ & 0.40 \\
\hline Franseen needle & 1 & & 1 & \\
\hline \multicolumn{5}{|l|}{ Location } \\
\hline Pancreas & 1 & & & \\
\hline Lymph node & $0.79(0.37-1.68)$ & 0.54 & & \\
\hline Submucosal tumor & $2.24(0.68-7.39)$ & 0.19 & & \\
\hline Others & $1.62(0.49-5.42)$ & 0.43 & & \\
\hline \multicolumn{5}{|l|}{ Access route } \\
\hline Transgastric & 1 & & 1 & \\
\hline Transduodenal & $0.52(0.32-0.85)$ & $<0.01$ & $0.50(0.31-0.82)$ & $<0.01$ \\
\hline Others & $1.55(0.36-6.72)$ & 0.56 & $1.42(0.33-6.19)$ & 0.64 \\
\hline \multicolumn{5}{|l|}{ Size of lesion } \\
\hline Small lesion & $0.53(0.32-0.86)$ & 0.01 & $0.52(0.31-0.86)$ & 0.01 \\
\hline Large lesion & 1 & & 1 & \\
\hline \multicolumn{5}{|l|}{ Physician } \\
\hline Expert & $0.83(0.51-1.35)$ & 0.45 & & \\
\hline Trainee & 1 & & & \\
\hline
\end{tabular}

CI, confidence interval; FNB, fine-needle biopsy; OR, odd ratio. 
Table 3. Characteristic of Study Lesions after Propensity Score Matching ( $n=482)$.

\begin{tabular}{|c|c|c|c|}
\hline & Menghini needle $(n=241)$ & Franseen needle $(n=241)$ & $p$-value \\
\hline & & & 0.91 \\
\hline \multirow{7}{*}{ Location, $n(\%)$} & $197(81.7 \%)$ & $193(80.1 \%)$ & \\
\hline & $79(40.1 \%)$ & $83(43.0 \%)$ & \\
\hline & $118(59.9 \%)$ & $110(57.0 \%)$ & \\
\hline & $20(8.3 \%)$ & $21(8.7 \%)$ & \\
\hline & $13(5.4 \%)$ & $17(7.1 \%)$ & \\
\hline & $2(15.4 \%)$ & $1(5.9 \%)$ & \\
\hline & $11(84.6 \%)$ & $16(94.1 \%)$ & \\
\hline Others & $11(4.6 \%)^{\mathrm{a})}$ & $10(4.1 \%)^{\mathrm{b})}$ & \\
\hline Access route, $n(\%)$ & & & 0.61 \\
\hline Transgastric & $141(58.5 \%)$ & $155(64.3 \%)$ & \\
\hline Transduodenal & $90(37.3 \%)$ & $77(32.0 \%)$ & \\
\hline Others & $10(4.1 \%)^{\mathrm{c})}$ & $9(3.7 \%)^{d)}$ & \\
\hline Size of lesion (mm), median (range) & $26(10-67)$ & $26(9-86)$ & 0.45 \\
\hline Number of punctures, median (range) & $2(1-6)$ & $2(1-6)$ & 0.33 \\
\hline Puncture length (mm), median (range) & $20(9-52)$ & $19(5-52)$ & 0.50 \\
\hline Experts/trainees, $n$ & $98 / 143$ & $77 / 164$ & 0.06 \\
\hline Tissue acquisition rate & $99.6 \%$ & $97.5 \%$ & 0.12 \\
\hline \multicolumn{4}{|l|}{ Diagnostic yield } \\
\hline Cytology only & $93.4 \%$ & $88.8 \%$ & 0.11 \\
\hline Histology only & $89.2 \%$ & $88.8 \%$ & 1.00 \\
\hline Histology plus cytology & $97.5 \%$ & $94.2 \%$ & 0.11 \\
\hline
\end{tabular}

${ }^{a)}$ Including intraperitoneal nodule 4, liver 3, adrenal grand 1, mediastinum 1, and gall bladder 2.

${ }^{\text {b) Including intraperitoneal nodule 8, liver 1, and mediastinum } 1 .}$

c) Transesophageal 10 .

d) Transesophageal 4, through the jejunum 3, and through the rectum 2.

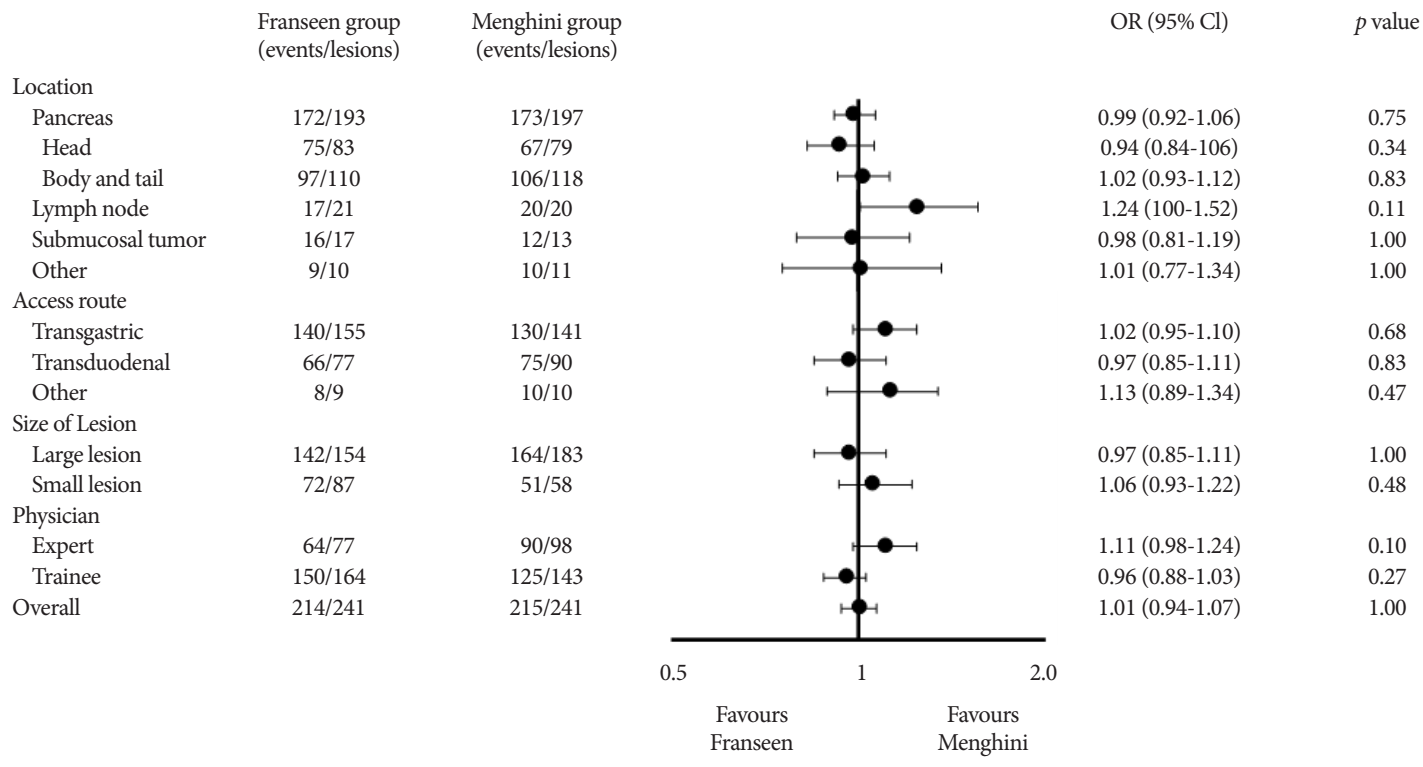

Fig. 3. Forest plot for subgroup analysis. Large lesion, $>2 \mathrm{~cm}$; Small lesion, $\leq 2 \mathrm{~cm}$. Cl, confidence interval; OR, odds ratio. 


\section{DISCUSSION}

We conducted a large, retrospective comparison of two FNB needles in 690 lesions. The puncture could be performed in almost all cases, and it was observed that both needles were easy to handle, even in cases of transduodenal access. The tissue acquisition rate was $98.4 \%$, the diagnosis rate of histology alone was $88.8 \%$, and the combined diagnosis rate of histology and cytology was $95.7 \%$, which were very good results. This high diagnostic yield could be obtained with a median of only two punctures using ROSE. The AE rate in this study was $1.5 \%$, which is similar to those mentioned in previous studies of EUS-FNA. Therefore, EUS-FNB can be considered as a safe procedure. ${ }^{29}$ Multivariate analysis showed that transduodenal access and small lesions were negative predictive factors for the diagnostic accuracy of histology, but the combined diagnostic rates of histology and cytology of each needle were relatively high (transduodenal access and small lesion, $94.9 \%$ and $91.1 \%$, respectively). After performing propensity score matching to adjust for background factors of these two needles, no clear difference was observed in the rate of tissue collection for histological diagnosis or the diagnostic yields of both needles. There have been no reports comparing the performance of these two FNB needles after adjusting for background factors, in such a large number of cases. In the subgroup analysis, there was no significant difference between the two needles. However, in case of lymph node lesions, $M$ needle was observed to have better results (OR, 1.24; 95\% CI, 1.00-1.52; $p=0.11$ ).

In this study, both the FNB needles had high diagnostic yields for obtaining a histological diagnosis. There are few reports on the diagnostic rate of FNB needles (include FNA needles in some cases) in small lesions, which is reported to be around $71 \%-82 \%$. However, it is not clear if this can be considered as the diagnostic rate for histology alone. ${ }^{30,31}$ In this study, $83.9 \%$ of the histological diagnoses and $91.1 \%$ of the combined diagnoses with histology and cytology were obtained, even for small lesions ( $\leq 2 \mathrm{~cm}$ ), which can be considered as promising results.

There are only a few reports comparing $\mathrm{M}$ needles with $\mathrm{F}$ needles: one retrospective study and one prospective study. ${ }^{32,33}$ In the retrospective study, FNB was performed for 34 lesions with $20 \mathrm{G}$ M needle, and 34 lesions with $22 \mathrm{G}$ F needle. The histological diagnosis rates with these two needles were equivalent ( $82 \%$ with $\mathrm{M}$ needle and $97 \%$ with F needle, $p=0.10){ }^{32}$ The same group reported a prospective study at UEGW 2019 , with 60 lesions punctured by both $20 \mathrm{G} \mathrm{M}$ and $22 \mathrm{G} \mathrm{F}$ needles. ${ }^{33} \mathrm{~A}$ histological diagnosis was achieved in $68 \%$ of the samples obtained with $\mathrm{M}$ needle and in $88 \%$ with $\mathrm{F}$ needle $(p=0.02)$. In addition, the authors reported that $\mathrm{F}$ needle was able to acquire longer tissue fragments than $\mathrm{M}$ needle. Howev$\mathrm{er}$, these studies were limited to a small number of pancreatic lesions; therefore, caution is required in the interpretation of their results. Regarding the length of the tissue fragment, the thickness of the needle is different between the $20 \mathrm{G}$ and $22 \mathrm{G}$ needles, and it is unknown whether the amount of collected tissue can be accurately evaluated.

In this study, propensity score matching was performed to adjust for background factors between the two groups. Even after adjustment, this was a large-scale study of 482 lesions, and there have been limited comparative reports on EUS-guided tissue acquisitions of this scale to date. ${ }^{11,34}$ As a result of propensity score matching, no clear differences were observed in the tissue collection rate and the diagnostic ability between $\mathrm{M}$ and F needles. Subgroup analysis also showed no clear difference between needles, but $\mathrm{M}$ needles tended to be slightly better for lymph node lesions. Since many lymph node lesions are soft lesions, the tissue can be easily cut by the forward bevel in the side hole, and the needle is as large as $20 \mathrm{G}$, so we speculate that $\mathrm{M}$ needle could potentially collect larger tissue samples. On the other hand, it was reported that the maneuverability of large-diameter needles deteriorates due to the bending of the scope, and it was expected that the diagnostic ability of $20 \mathrm{G}$ M needle might decrease in cases of transduodenal access or pancreatic head lesions. ${ }^{23,35,36}$ However, no significant difference was observed between the two groups for either the pancreatic head lesions or transduodenal access in our study.

There are some limitations in this study. First, this is a retrospective study of a single center. However, this study includes a large number of cases extracted from a prospectively accumulated database, and an analysis after propensity score matching was also performed with a large number of cases. Second, not all FNB needles available in the world were evaluated in this study. The FNB needle with a fork tip is widely used. However, this needle has not yet been introduced in Japan. There are some reports comparing Fork-tip needle and F needle, yet the superiority of one over the other in terms of diagnostic accuracy remains controversial. ${ }^{37,38}$ Therefore, it is necessary to compare these three needles in future studies. Third, the amounts of tissue collected cannot be compared objectively. Moreover, it is necessary to further study whether the sample enables the genetic panel test that is currently attracting attention. Fourth, in the forest plot, there were few tumors other than pancreatic lesions; therefore, the location may have affected the results. Finally, not only experts but also trainees were included as operators. While there was a higher tendency for experts to be involved in the $\mathrm{M}$ group after propensity score matching, it did not influence the histological diagnostic accuracy in multi- 
variate analysis. This is similar to what is encountered in actual clinical practice and this result can thus be generalized.

In conclusion, we conducted a comparative study on the efficacy and safety of two FNB needles in a large number of cases. Unlike previous reports, both $\mathrm{M}$ and $\mathrm{F}$ needles proved to have good diagnostic abilities and safety, with no clear difference in the performance of these two needles.

Conflicts of Interest

The authors have no potential conflicts of interest.

Funding

None.

Author Contributions

Conceptualization: Takafumi Mie, Takashi Sasaki, Ryo Kanata, Masato Matsuyama, Naoki Sasahira

Data curation: TM, RK, Takaaki Furukawa, Tsuyoshi Takeda, Akiyoshi Kasuga

Formal analysis: TM, TS, RK, TT

Investigation: TM, RK, TF, TT, AK

Methodology: TM, TS, RK, TT

Project administration: Masato Ozaka, NS

Supervision: MO, NS

Writing-original draft: TM

Writing-review\&editing: TS, TT, NS

\section{ORCID}

Takafumi Mie:

Takashi Sasaki:

Ryo Kanata:

Takaaki Furukawa:

Tsuyoshi Takeda:

Akiyoshi Kasuga:

Masato Ozaka:

Naoki Sasahira:
Masato Matsuyama:

\begin{abstract}
https://orcid.org/0000-0002-7561-5558 https://orcid.org/0000-0001-7109-9835 https://orcid.org/0000-0001-7544-8609 https://orcid.org/0000-0002-9746-6776 https://orcid.org/0000-0002-0786-8166 https://orcid.org/0000-0002-2234-1135 https://orcid.org/0000-0003-3604-1624 https://orcid.org/0000-0002-5666-1970 https://orcid.org/0000-0002-0672-7214
\end{abstract}

\section{REFERENCES}

1. Ryozawa S, Kitoh H, Gondo T, et al. Usefulness of endoscopic ultrasound-guided fine-needle aspiration biopsy for the diagnosis of pancreatic cancer. J Gastroenterol 2005;40:907-911.

2. Yasuda I, Tsurumi H, Omar S, et al. Endoscopic ultrasound-guided fine-needle aspiration biopsy for lymphadenopathy of unknown origin. Endoscopy 2006;38:919-924.

3. Inoue T, Okumura F, Sano H, et al. Impact of endoscopic ultrasound-guided fine-needle biopsy on the diagnosis of subepithelial tumors: a propensity score-matching analysis. Dig Endosc 2019;31:156163.

4. Kamata K, Kitano M, Yasukawa S, et al. Histologic diagnosis of pancreatic masses using 25-gauge endoscopic ultrasound needles with and without a core trap: a multicenter randomized trial. Endoscopy 2016;48:632-638.

5. Iwashita T, Yasuda I, Doi S, et al. Use of samples from endoscopic ultrasound-guided 19-gauge fine-needle aspiration in diagnosis of autoimmune pancreatitis. Clin Gastroenterol Hepatol 2012;10:316-322.

6. Lee YN, Moon JH, Kim HK, et al. Core biopsy needle versus standard aspiration needle for endoscopic ultrasound-guided sampling of solid pancreatic masses: a randomized parallel-group study. Endoscopy 2014;46:1056-1062.

7. Lee BS, Cho CM, Jung MK, Jang JS, Bae HI. Comparison of histologic core portions acquired from a core biopsy needle and a conventional needle in solid mass lesions: a prospective randomized trial. Gut Liver 2017;11:559-566.

8. Sterlacci W, Sioulas AD, Veits L, et al. 22-gauge core vs 22-gauge aspiration needle for endoscopic ultrasound-guided sampling of abdominal masses. World J Gastroenterol 2016;22:8820-8830.

9. Fujie $\mathrm{S}$, Ishiwatari $\mathrm{H}$, Sasaki K, et al. Comparison of the diagnostic yield of the standard 22-gauge needle and the new 20-gauge forward-bevel core biopsy needle for endoscopic ultrasound-guided tissue acquisition from pancreatic lesions. Gut Liver 2019;13:349-355.

10. Nishioka N, Ogura T, Kurisu Y, et al. Prospective histological evaluation of a $20 \mathrm{G}$ core trap with a forward-cutting bevel needle for EUS-FNA of pancreatic lesions. Surg Endosc 2018;32:4125-4131.

11. van Riet PA, Larghi A, Attili F, et al. A multicenter randomized trial comparing a 25-gauge EUS fine-needle aspiration device with a 20-gauge EUS fine-needle biopsy device. Gastrointest Endosc 2019;89:329-339.

12. Fabbri C, Fornelli A, Fuccio L, et al. High diagnostic adequacy and accuracy of the new 20G procore needle for EUS-guided tissue acquisition: results of a large multicentre retrospective study. Endosc Ultrasound 2019;8:261-268.

13. Armellini E, Manfrin E, Trisolini E, et al. Histologic retrieval rate of a newly designed side-bevelled 20G needle for EUS-guided tissue acquisition of solid pancreatic lesions. United European Gastroenterol J 2019;7:96-104.

14. Mita N, Iwashita T, Uemura S, et al. Endoscopic ultrasound-guided fine-needle biopsy using 22-gauge Franseen needle for the histological diagnosis of solid lesions: a multicenter prospective pilot study. Dig Dis Sci 2020;65:1155-1163.

15. Bang JY, Hebert-Magee S, Hasan MK, Navaneethan U, Hawes R, Varadarajulu S. Endoscopic ultrasonography-guided biopsy using a Franseen needle design: initial assessment. Dig Endosc 2017;29:338-346.

16. Kandel P, Tranesh G, Nassar A, et al. EUS-guided fine-needle biopsy sampling using a novel fork-tip needle: a case-control study. Gastrointest Endosc 2016;84:1034-1039.

17. Matsuno J, Ogura T, Kurisu Y, et al. Prospective comparison study of franseen needle and standard needle use for pancreatic lesions under EUS guidance. Endosc Ultrasound 2019;8:412-417.

18. Mukai S, Itoi T, Yamaguchi H, et al. A retrospective histological comparison of EUS-guided fine-needle biopsy using a novel franseen needle and a conventional end-cut type needle. Endosc Ultrasound 2019;8:5057.

19. El H, II, Wu H, Reuss S, et al. Prospective assessment of the performance of a new fine-needle biopsy device for EUS-guided sampling of solid lesions. Clin Endosc 2018;51:576-583.

20. Renelus BD, Jamorabo DS, Boston I, Briggs WM, Poneros JM. Endoscopic ultrasound-guided fine-needle biopsy needles provide higher diagnostic yield compared to endoscopic ultrasound-guided fine-needle aspiration needles when sampling solid pancreatic lesions: a meta-analysis. Clin Endosc 2021;54:261-268.

21. Gleeson FC, Levy MJ, Roden AC, et al. EUS fine-needle pancreatic core biopsy can determine eligibility for tumor-agnostic immunotherapy. Endosc Int Open 2018;6:E1278-E1282.

22. Muniraj T, Aslanian HR. New developments in endoscopic ultrasound tissue acquisition. Gastrointest Endosc Clin N Am 2017;27:585-599.

23. Conti CB, Cereatti F, Grassia R. Endoscopic ultrasound-guided sampling of solid pancreatic masses: the fine-needle aspiration or fine-needle biopsy dilemma. Is the best needle yet to come? World J Gastrointest Endosc 2019;11:454-471.

24. Bang JY, Magee SH, Ramesh J, Trevino JM, Varadarajulu S. Randomized trial comparing fanning with standard technique for endoscopic ultra- 
sound-guided fine-needle aspiration of solid pancreatic mass lesions. Endoscopy 2013;45:445-450.

25. Nakai Y, Isayama $\mathrm{H}$, Chang KJ, et al. Slow pull versus suction in endoscopic ultrasound-guided fine-needle aspiration of pancreatic solid masses. Dig Dis Sci 2014;59:1578-1585.

26. Cotton PB, Eisen GM, Aabakken L, et al. A lexicon for endoscopic adverse events: report of an ASGE workshop. Gastrointest Endosc 2010;71:446-454.

27. Kanda Y. Investigation of the freely available easy-to-use software 'EZR' for medical statistics. Bone Marrow Transplant 2013;48:452-458.

28. Sasaki T, Kanata R, Sasahira N. Needle fracture during endoscopic ultrasound-guided fine-needle aspiration using a needle with a side hole. Endosc Int Open 2018;6:E553-E557.

29. Yoshinaga S, Itoi T, Yamao K, et al. Safety and efficacy of endoscopic ultrasound-guided fine-needle aspiration for pancreatic masses: a prospective multicenter study. Dig Endosc 2020;32:114-126.

30. Hwang CY, Lee SS, Song TJ, et al. Endoscopic ultrasound guided fine-needle aspiration biopsy in diagnosis of pancreatic and peripancreatic lesions: a single center experience in Korea. Gut Liver 2009;3:116 121.

31. Fabbri C, Luigiano C, Maimone A, et al. Endoscopic ultrasound-guided fine-needle biopsy of small solid pancreatic lesions using a 22-gauge needle with side fenestration. Surg Endosc 2015;29:1586-1590.

32. Karsenti D, Tharsis G, Zeitoun JD, et al. Comparison of 20-gauge Pro- core and 22-gauge Acquire needles for EUS-FNB of solid pancreatic masses: an observational study. Scand J Gastroenterol 2019;54:499-505.

33. Karsenti D, Palazzo L, Perrot B, et al. Multicenter randomized controlled trial (RCT) comparing the histological material quantity obtained by endoscopic ultrasound fine-needle biopsy (EUS-FNB) of pancreatic masses with two „biopsy“ needles: the 20-gauge Procore (Cook) and the 22-gauge Acquire (Boston Scientific). United European Gastroenterol J 2019;7(8 Suppl):28.

34. Bang JY, Kirtane S, Krall K, et al. In memoriam: fine-needle aspiration, birth: fine-needle biopsy: the changing trend in endoscopic ultrasound-guided tissue acquisition. Dig Endosc 2019;31:197-202.

35. Karadsheh Z, Al-Haddad M. Endoscopic ultrasound-guided fine-needle aspiration needles: which one and in what situation? Gastrointest Endosc Clin N Am 2014;24:57-69.

36. Vilmann P, Seicean A, Săftoiu A. Tips to overcome technical challenges in EUS-guided tissue acquisition. Gastrointest Endosc Clin N Am 2014;24:109-124

37. Abdelfatah MM, Grimm IS, Gangarosa LM, Baron TH. Cohort study comparing the diagnostic yields of 2 different EUS fine-needle biopsy needles. Gastrointest Endosc 2018;87:495-500.

38. Bang JY, Hebert-Magee S, Navaneethan U, Hasan MK, Hawes R, Varadarajulu S. Randomized trial comparing the Franseen and Fork-tip needles for EUS-guided fine-needle biopsy sampling of solid pancreatic mass lesions. Gastrointest Endosc 2018;87:1432-1438. 